\title{
MYEOV Regulation in Cancer
}

\author{
Shivani Golem* \\ Department of Pathology and Laboratory Medicine, University of Kansas Medical \\ Center, USA
}

*Corresponding author: Shivani Golem, Department of Pathology and Laboratory Medicine, University of Kansas Medical Center, Kansas City, Kansas, USA, Tel: 913945-6002; Email: sgolem@kumc.edu

Abbreviations: MYEOV: Myeloma Overexpressed; NSCLC: Non-Small Cell Lung Cancer; CCND1: Cyclin D1; IGH: Immunoglobulin Heavy; ceRNA: Competing endogenous RNA.

\section{Editorial}

Myeloma Overexpressed (MYEOV), a gene at 11q13 chromosome break point and most commonly referred as a gene located $360 \mathrm{~kb}$ centromeric to CCND1 gene is overexpressed and involved in tumor genesis in number of cancers including breast cancer, gastric cancer, esophageal squamous cell carcinoma, colon cancer, nonsmall cell lung cancer (NSCLC) and in multiple myeloma. Studies on MYEOV gene have shown both CCND1/IGH dependent and independent regulation of expression of MYEOV. In this special issue, a collection of five articles contribute to the underlying of mechanisms of MYEOV regulation.

The paper by Janssen JW, et al. [1] observed that in a subset of multiple myeloma cell lines both CCND1 and MYEOV are co-activated by $\mathrm{t}(11 ; 14)$ (q13;q32); and the Immunoglobulin heavy (IGH) region involved were either the 5' $\mathrm{E} \mu$ or the telomeric 3' $\mathrm{E} \alpha$ enhancers, which juxtaposed to the MYEOV. The paper by Janssen JW, et al. [2] presents amplification of MYEOV along with CCND1 in esophageal squamous cell carcinomas. However, in few of the cell lines assessed in this study, MYEOV was epigenetically inactivated, explaining the discrepancy of MYEOV amplification without high MYEOV expression.

The paper by de Almeida RA, et al. [3] proposed MYEOV gene translation is tightly controlled by upstream AUGs in the 5'-untranslated region of MYEOV gene. The paper by Coccaro $\mathrm{N}$, et al. [4] demonstrated that IGH variable region juxtaposition to MYEOV and CCND1 was responsible for both of these genes expression in a case of primary plasma cell leukemia with $\mathrm{t}(11 ; 14)(\mathrm{q} 13 ; \mathrm{q} 32)$.

The paper by Fang L, et al. [5] showed that MYEOV competing endogenous RNA (ceRNA) or MYEOV transcript is upregulated in NSCLC, and played a role in the invasion and metastasis of NSCLC. The MYEOV ceRNA showed its tumorigenic activity in a miR-30c-2-3p binding-dependent manner, and by activating TGF- $\beta$ signaling. This mode of action of MYEOV was independent of its protein-coding capacity. Taken together, these articles in this special issue show the spectrum of mechanism by which MYEOV gene is regulated in different cancer types. This may provide the understanding and need for assessing MYEOV gene regulation using different mechanistic approaches when MYEOV gene alteration is detected at DNA, RNA or protein level.

\section{References}

1. Janssen JW, Vaandrager JW, Heuser T, Jauch A, Kluin PM, et al. (2000) Concurrent activation of a novel putative transforming gene, myeov, and cyclin D1 in a subset of multiple myeloma cell lines with $\mathrm{t}(11 ; 14)(q 13 ; q 32)$. Blood 95(8): 2691-2698.

2. Janssen JW, Imoto I, Inoue J, Shimada Y, Ueda M, et al. (2002) MYEOV, a gene at 11q13, is coamplified with CCND1, but epigenetically inactivated in a subset of esophageal squamous cell carcinomas. J Hum Genet 47(9): 460-464.

3. de Almeida RA, Heuser T, Blaschke R, Bartram CR, Janssen JW (2006) Control of MYEOV protein 
synthesis by upstream open reading frames. J Biol Chem 281(2): 695-704.

4. Coccaro N, Tota G, Anelli L, Zagaria A, Casieri P, et al. (2016) MYEOV gene overexpression in primary plasma cell leukemia with $\mathrm{t}(11 ; 14)(\mathrm{q} 13 ; \mathrm{q} 32)$. Oncol Lett 12(2): 1460-1464.
5. Fang L, Wu S, Zhu X, Cai J, Wu J, et al. (2018) MYEOV functions as an amplified competing endogenous RNA in promoting metastasis by activating TGF- $\beta$ pathway in NSCLC. Oncogene 38(6): 896-912. 